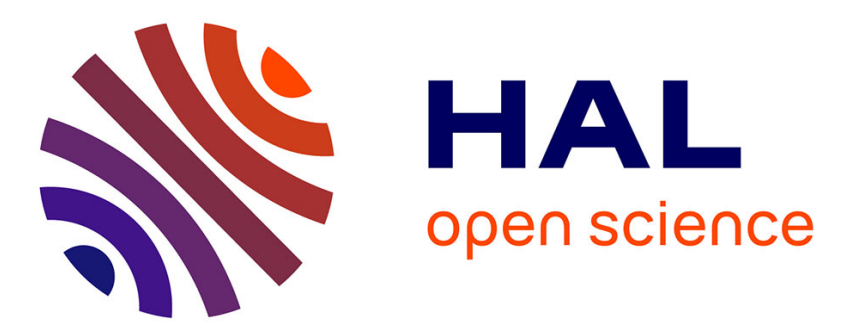

\title{
Emergence of AcrAB-mediated tigecycline resistance in a clinical isolate of during ciprofloxacin treatment
}

Michael Hornsey, Matthew J. Ellington, Michel Doumith, Geoff Scott, David M. Livermore, Neil Woodford

\section{- To cite this version:}

Michael Hornsey, Matthew J. Ellington, Michel Doumith, Geoff Scott, David M. Livermore, et al.. Emergence of AcrAB-mediated tigecycline resistance in a clinical isolate of during ciprofloxacin treatment. International Journal of Antimicrobial Agents, 2010, 35 (5), pp.478. 10.1016/j.ijantimicag.2010.01.011 . hal-00578293

\section{HAL Id: hal-00578293 \\ https://hal.science/hal-00578293}

Submitted on 19 Mar 2011

HAL is a multi-disciplinary open access archive for the deposit and dissemination of scientific research documents, whether they are published or not. The documents may come from teaching and research institutions in France or abroad, or from public or private research centers.
L'archive ouverte pluridisciplinaire HAL, est destinée au dépôt et à la diffusion de documents scientifiques de niveau recherche, publiés ou non, émanant des établissements d'enseignement et de recherche français ou étrangers, des laboratoires publics ou privés. 


\section{Accepted Manuscript}

Title: Emergence of AcrAB-mediated tigecycline resistance in a clinical isolate of Enterobacter cloacae during ciprofloxacin treatment

Authors: Michael Hornsey, Matthew J. Ellington, Michel Doumith, Geoff Scott, David M. Livermore, Neil Woodford

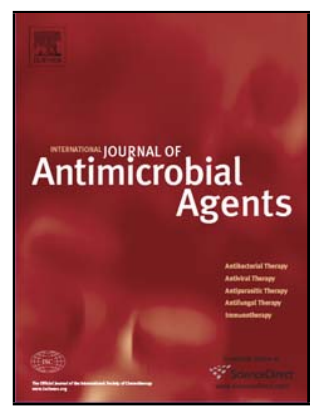

PII:

DOI:

Reference:

S0924-8579(10)00056-7

ANTAGE 3235

To appear in: International Journal of Antimicrobial Agents

Received date: $\quad$ 5-1-2010

Accepted date: $\quad$ 6-1-2010

Please cite this article as: Hornsey M, Ellington MJ, Doumith M, Scott G, Livermore DM, Woodford N, Emergence of AcrAB-mediated tigecycline resistance in a clinical isolate of Enterobacter cloacae during ciprofloxacin treatment, International Journal of Antimicrobial Agents (2008), doi:10.1016/j.ijantimicag.2010.01.011

This is a PDF file of an unedited manuscript that has been accepted for publication. As a service to our customers we are providing this early version of the manuscript. The manuscript will undergo copyediting, typesetting, and review of the resulting proof before it is published in its final form. Please note that during the production process errors may be discovered which could affect the content, and all legal disclaimers that apply to the journal pertain. 


\section{Emergence of AcrAB-mediated tigecycline resistance in a clinical} isolate of Enterobacter cloacae during ciprofloxacin treatment

3

4 Michael Hornsey $^{1 *}$, Matthew J. Ellington ${ }^{1 \dagger}$, Michel Doumith ${ }^{1}$, Geoff $\operatorname{Scott}^{2}$, David M.

5

6

7

8

9

10

11

12

13

14

15

16

17

18

19

20

21

22

23

24

25 3 4

\section{(8)} 9 2 3

${ }^{1}$ Antibiotic Resistance Monitoring and Reference Laboratory, Health Protection Agency Centre for Infections, London NW9 5EQ UK; ${ }^{2}$ University College London Hospitals, Windeyer Institute, London W1T 4JF UK

${ }^{\dagger}$ Present address: Clinical Microbiology and Public Health Laboratory, Health Protection Agency, Addenbrooke's Hospital, Hills Road, Cambridge, CB2 0QQ UK

\section{* Corresponding author.}

Tel: +44-20-8327-6176; Fax: +44-20-8327-6264; E-mail:

michael.hornsey@hpa.org.uk

1 Keywords: RND pumps; glycylcycline; Enterobacteriaceae 


\section{6}

27 6 7 0

\section{Abstract}

Tigecycline resistance remains rare amongst Enterobacteriaceae in the UK, as elsewhere, but has been associated with up-regulation of the AcrAB efflux system. Using isolates of an Enterobacter cloacae strain that developed tigecycline resistance in vivo during ciprofloxacin therapy and laboratory-selected mutants, we investigated the role of this pump and the global regulator RamA in this resistance. Laboratory mutants were selected from a susceptible clinical isolate in vitro by exposure to increasing concentrations of tigecycline. Expression of the acrAB operon and $\operatorname{ram} A$ gene was monitored by real-time RT-PCR. Overexpression of $\operatorname{ramA}$ was achieved using the $\mathrm{pBAD}$ expression vector, whilst insertional inactivation of $\operatorname{acr} B$ with a gentamicin resistance cassette was achieved with the bacteriophage $\lambda$ Red recombination system. Increased tigecycline MICs in the clinical isolate and a laboratory mutant were associated with increases in $\operatorname{acr} A B$ and $\operatorname{ram} A$ transcripts. Induction of increased ramA expression resulted in increased acrAB expression, whilst insertional inactivation of $a c r B$ restored full susceptibility to tigecycline.

Treatment with ciprofloxacin - a substrate of AcrAB in E. cloacae - possibly selected for cross-resistance to tigecycline as a result of RamA-mediated AcrAB up-regulation. 3

(1)
8 9 


\section{Introduction}

52 Enterobacter cloacae is the most important nosocomial pathogen in the genus

53 Enterobacter and is of increasing concern to clinicians owing to the many isolates that

54 are multi-drug resistant. Tigecycline has good in vitro activity against E. cloacae and

55 is a welcome addition to the armamentarium (1), however, E. cloacae is the least

56 susceptible member of the genus to this compound (2) and cases of emerging resistance have been described (3-5). Where their mechanisms have been investigated, up-regulation of the RND efflux system AcrAB and the global regulator RamA have been implicated (5).

61 We investigated the role of these components in an E. cloacae strain that developed tigecycline resistance during ciprofloxacin treatment and in vitro, under tigecycline pressure.

\subsection{Case report}

In 2006, a patient presented with a ten-day history of progressively increasing back pain, high fever $\left(40^{\circ} \mathrm{C}\right)$ and swelling over the spine. He had had two previous operations for stabilisation of a lumbar spinal fracture, 20 months, followed by a revision 16 months, prior to this event. An aspirate revealed two colony variants of Enterobacter cloacae which varied in their antibiotic susceptibility patterns. Both appeared sensitive to ciprofloxacin and the initial regimen was changed to ciprofloxacin (400mg 12 hourly iv) on day four after admission. A definitive surgical procedure was performed three days later and one litre of pus and all the metalwork

74 (eight screws and plates) were removed. The same organisms were isolated again but 
76 resistant to ciprofloxacin (here called isolate TGC-R) and the other remaining

77 susceptible (isolate TGC-S). This pair of isolates was referred to the Health Protection

78 Agency's Antibiotic Resistance Monitoring and Reference Laboratory, where the

79 ciprofloxacin-resistant isolate was found also to be resistant to tigecycline.

80 Ciprofloxacin was subsequently replaced with meropenem after eight days and the

81 patient slowly recovered after several months of carbapenem therapy. The original 82 source of the infection was not established.

83

84 


\section{Materials and Methods}

2.1. Bacterial isolates, identification, and growth conditions

87

88

89

90

91

92

93

94

95

96

97

98

99

100

101

102

103

104

105

106

107

108

The organisms were clinical isolates, laboratory-selected mutants, or gene knockout mutants of E. cloacae (Table 1). The clinical isolates, designated TGC-S and TGC-R, were identified by API20E (bioMérieux, Marcy l'Etoile, France). PFGE was performed on XbaI-digested genomic DNA to determine isolate relatedness. Isolates were propagated in Luria-Bertani (LB) or Iso-Sensitest (Oxoid, Basingstoke, UK) broth or agar at $37^{\circ} \mathrm{C}$.

\subsection{Antimicrobial susceptibility testing}

MICs of tigecycline, tetracycline, minocycline, ciprofloxacin, chloramphenicol, meropenem, and cefpirome were determined by agar dilution on Iso-Sensitest agar according to BSAC guidelines or by E-test (AB Biodisk, Solna, Sweden) used on IsoSensitest agar and MICs were interpreted using BSAC breakpoints (6).

\subsection{Laboratory-selection of mutants}

Clinical isolate TGC-S was passaged with serially increasing concentrations of tigecycline in Iso-Sensitest broth, beginning at half the MIC $(0.25 \mathrm{mg} / \mathrm{L})$ and the tigecycline concentration doubled every 24 hours until there was no further growth. MICs for mutants were determined daily by E-test. PFGE profiles of the final mutant (TGC-S7) and all intermediates were compared with that of isolate TGC-S in order to confirm parentage. 
110 Gel electrophoresis and restriction enzyme digestion were performed as previously

111 described (7). Genomic DNA was isolated using the Wizard Genomic DNA

112 Purification Kit (Promega, Southampton, UK) and was used as the template for PCR.

113 Plasmids were isolated from $\alpha$-select Escherichia coli (Bioline, London, UK) using

114 the PureYield Plasmid Miniprep System (Promega). PCR products were purified

115 using the Geneclean Turbo for PCR (MP Biomedicals, Strasbourg, France). DNA

116 transformations were performed by electroporation $(1.8 \mathrm{kV}, 25 \mu \mathrm{F}, 200 \Omega)$ using the

117 Gene Pulser system (Bio-Rad, Hemel Hempstead, UK). Nucleotide sequences were

118 determined with a CEQ 8000 Genetic Analysis System automatic sequencer

119 (Beckman Coulter, High Wycombe, UK). The nucleotide sequence data reported here

120 have been deposited with GenBank with the accession numbers GU180677 and

\section{$121 \quad \underline{\text { GU180678. }}$}

\subsection{Real-time RT-PCR}

124 Expression of the $a c r A B$ operon and the $\operatorname{ram} A$ gene was monitored by real-time RT-

125 PCR and quantified relative to that of the RNA polymerase $\beta$ subunit gene, rpoB,

126 using oligonucleotide primers specific for $\operatorname{acrB}$ (forward, 5'-CGA TAA CCT GAT

127 GTA CAT GTC C-3'; reverse, 5'-CCG ACA ACC ATC AGG AAG CT-3'), ramA

128 (forward, 5'-CCG TTA CGC ATC GAA GAG AT-3'; reverse, 5'-CCC AGA CTT

129 TCG CCT TTG TA-3'), and rpoB (forward, 5'-AAG GCG AAT CCA GCT TGT TCA

130 GC-3'; reverse, 5'-TGA CGT TGC ATG TTC GCA CCC ATC A-3') to amplify

131 products of 185,92 , and $147 \mathrm{bp}$, respectively. 
133 DNase-treated RNA template was prepared from overnight cultures using the RNeasy

134 Mini Kit (Qiagen, Crawley, UK) and RT-PCR was undertaken using the QuantiFast

135 SYBR Green RT-PCR Kit (Qiagen) on a LightCycler 2.0 Real-Time PCR System

136 (Roche, Burgess Hill, UK) with 10 ng RNA and protocols supplied by the

137 manufacturer. Normalised expression levels of the target gene (relative to rpoB) were

138 calculated using the $2^{-\Delta \Delta C T}$ method (8). Reactions were performed in triplicate using

139 template from separate RNA extractions.

140

\subsection{Overexpression of ramA in clinical isolate TGC-S}

142 The prokaryotic expression vector pBAD (Invitrogen, Paisley, UK) was modified to

143 replace the unique $N c o$ I restriction site with a unique SmaI site, yielding plasmid

144 pBADS. A 1138 bp DNA fragment containing the kanamycin resistance cassette from

145 pCR2.1 (Invitrogen) was then amplified by PCR using the proofreading Pfu DNA

146 polymerase (Promega) (forward primer, 5'-GCT TAC ATG GCG ATA GCT AGA

147 CT-3'; reverse primer, 5'-AGT TCG ATG TAA CCC ACT CGT GC-3'), and cloned

148 into EcoRV-digested pBADS to give the final vector pBADKM.

149

150 The $\operatorname{ramA}$ gene was amplified by PCR from clinical isolate TGC-R using Pfu DNA

151 polymerase and the following primer pair: forward, 5'-ATG ACG ATT TCC GCT

152 CAG GTC-3'; reverse, 5'-TGA AGC TTT CAG TGC GTC CGA CTA TGG TTT TC-

$153 \quad 3$ '. The resulting 350-bp product was digested with HindIII and directionally cloned

154 into SmaI/HindIII-digested pBADKM to produce plasmid pBADKM-R, which was

155 transformed into isolate TGC-S for the ramA overexpression studies; vector

156 pBADKM was used as a negative control. 
157 LB broth $(10 \mathrm{ml})$ containing $50 \mathrm{mg} / \mathrm{L}$ kanamycin was seeded with $100 \mu \mathrm{l}$ of an

158 overnight culture of TGC-S harbouring either pBADKM or pBADKM-R and

159 incubated with shaking $(225 \mathrm{rpm})$ at $37^{\circ} \mathrm{C}$ to mid-log phase $\left(\mathrm{OD}_{600}=0.5\right)$. Expression

160 of $\operatorname{ramA}$ was then induced for 4 hours by the addition of L-arabinose to final

161 concentrations of $0.2,0.02,2 \times 10^{-3}$, and $2 \times 10^{-4} \% \mathrm{w} / \mathrm{v}$. RNA extractions were then

162 performed and expression of $a c r A B$ was monitored by real-time RT-PCR.

163

\subsection{Insertional inactivation of acrB}

165 The $\operatorname{acr} B$ gene was inactivated by the insertion of a gentamicin resistance cassette

166 using methods described by Pérez et al. (9) with some modifications. The helper

167 plasmid pKOBEG (10) was introduced into TGC-R and transformants selected on LB

168 agar plates containing $100 \mathrm{mg} / \mathrm{L}$ chloramphenicol during overnight incubation at

$16930^{\circ} \mathrm{C}$. A single such transformant was made electrocompetent as follows: $20 \mathrm{ml}$ of

170 modified LB broth (without $\mathrm{NaCl}$ ) containing $100 \mathrm{mg} / \mathrm{L}$ chloramphenicol was seeded

171 with $200 \mu \mathrm{l}$ of an overnight culture of TGC-R harbouring pKOBEG and incubated at

$17230^{\circ} \mathrm{C}$ with shaking $(200 \mathrm{rpm})$. When the $\mathrm{OD}_{600}$ reached 0.2 the phage $\lambda$ Red genes

173 encoded by pKOBEG were induced by the addition of L-arabinose to a final

174 concentration of $0.2 \% \mathrm{w} / \mathrm{v}$. When the $\mathrm{OD}_{600}$ reached 1.0 the culture was centrifuged

175 at $4000 \mathrm{~g}$ at $4^{\circ} \mathrm{C}$ for 10 minutes, the supernatant removed and the pellet re-suspended

176 in $40 \mathrm{ml}$ of cold sterile water and re-centrifuged as before. The final pellet was

177 suspended in $90 \mu \mathrm{l}$ of $15 \%$ sterile glycerol.

178

179 An internal 1558-bp fragment of the $a c r B$ gene was amplified by the Expand High

180 Fidelity Plus PCR system (Roche) from TGC-R genomic DNA using primers 5'-CAT

181 CCA GCA CTT TCT GCG TA-3' (forward) and 5'-CGA TAA CCT GAT GTA CAT 
182 GTC C-3' (reverse) and cloned into pCR2.1 to generate plasmid pCRBK-1. The

183 gentamicin resistance cassette from cloning vector pBBR1MCS-5 was amplified by

184 PCR using Pfu DNA polymerase and primers 5'-TGG CGG CGT TGT GAC AAT

185 TTA C-3' (forward), and 5'-TGG ACG CAC ACC GTG GAA ACG G-3' (reverse)

186 (11). The resulting 854-bp fragment was cloned into NruI-digested pCRBK-1 (within

187 the $a c r B$ fragment) to generate pCRBK-2. The linear DNA fragment employed to

188 interrupt $a c r B$ was then amplified from pCRBK-2 using the internal $a c r B$ fragment

189 primers detailed above and the Expand High Fidelity Plus PCR system. The resulting

190 PCR product was introduced into TGC-R::pKOBEG, and transformants were selected

191 on LB agar plates containing $10 \mathrm{mg} / \mathrm{L}$ gentamicin. Chromosomal integration of the

192 gentamicin resistance cassette was confirmed by PCR and sequencing across the

193 insertion site.

194

195 


\section{Results and Discussion}

197 MICs of ciprofloxacin and tigecycline were eight-fold higher for the clinical isolate

198 TGC-R (4 mg/L for both compounds) than for TGC-S (both $0.5 \mathrm{mg} / \mathrm{L}$; Table 1).

199 Although this clinical pair did not have identical PFGE profiles, analysis of the

200 intermediate laboratory-selected mutants derived from TGC-S showed an analogous

201 transition from the tigecycline-susceptible to the tigecycline-resistant profile;

202 moreover, the PFGE profile of the final mutant TGC-S7 (ciprofloxacin MIC $4 \mathrm{mg} / \mathrm{L}$;

203 tigecycline MIC $32 \mathrm{mg} / \mathrm{L}$ ) was indistinguishable from that of clinical isolate TGC-R

204 (Figure 1). MICs of chloramphenicol, minocycline, and tetracycline also were raised

205 for TGC-R and TGC-S7 relative to TGC-S, whereas those of $\beta$-lactams were

206 unaffected, although that of cefpirome was high already $(8 \mathrm{mg} / \mathrm{L})$, possibly reflecting

207 AmpC-activity, as based on interpretive reading of wider antibiogram data (data not

208 shown).

209

210 Expression of $a c r A B$ and $\operatorname{ram} A$ was analysed by real-time RT-PCR. Relative to the

211 susceptible isolate, TGC-S expression of $a c r A B$ and $\operatorname{ram} A$ was increased four- and

212 276-fold, respectively, in isolate TGC-R and nine- and 407-fold, respectively, in the

213 laboratory mutant TGC-S7.

215 Keeney et al. have previously demonstrated an association between elevated

216 tigecycline MICs and overexpression of acrAB and ramA in E. cloacae (4). However,

217 no previous experimental evidence has placed $\operatorname{acr} A B$ definitively in the RamA

218 regulon. In order to investigate this aspect, $\operatorname{ramA}$ from isolate TGC-R was

219 overexpressed in isolate TGC-S using the pBAD expression vector. Induction of

$220 \operatorname{ramA}$ expression by increasing the L-arabinose concentration resulted in increased 
$\operatorname{acr} A B$ expression (Figure 2$)$. When $\operatorname{ram} A$ was induced with a relatively high $(0.2 \%$ w/v) concentration of L-arabinose, $a c r A B$ was expressed at a higher level than in isolate TGC-R, for which the tigecycline MIC was $4 \mathrm{mg} / \mathrm{L}$ (relative $a c r A B$ expression

$224=7.75$ versus 4.43 for TGC-R). Comparison of the nucleotide sequence of ramA 225 (partial coding region) in isolates TGC-S and TGC-R revealed a His-95 $\rightarrow$ Arg 226 substitution in TGC-R, although this seems unlikely to be critical as it was not present in TGC-S7.

The $a c r B$ gene in isolate TGC-R was disrupted by insertional inactivation using the helper plasmid pKOBEG and a linear DNA fragment containing a gentamicin

231 resistance cassette flanked by regions complementary to the target gene. This restored 232 full susceptibility to tigecycline, ciprofloxacin, chloramphenicol, and resulted in a 233 large reduction in the MICs of minocycline ( $\geq 64$-fold) and tetracycline (16-fold)

234 (Table 1). These data confirm that up-regulation of AcrAB, mediated by RamA 235 activation, was the factor conferring tigecycline resistance in E. cloacae.

237 To our knowledge, this is the first report of emergence of resistance to tigecycline 238 associated with ciprofloxacin therapy, rather than tigecycline therapy. Ciprofloxacin is 239 known to be a substrate of AcrAB in E. cloacae (9) and it is possible that exposure to 240 ciprofloxacin selected for AcrAB up-regulation, which resulted in cross-resistance to 241 tigecycline. The potential to select cross-resistance to new antimicrobials such as 242 tigecycline through the use of established front-line therapies is of concern in the 243 clinic. 


\section{Acknowledgements}

247 We thank A. Pérez for providing plasmid pKOBEG.

\section{Declarations}

252 Funding: This work was supported by an educational grant from Wyeth.

253 Competing interests: D. M. L. has (i) received research grants from Wyeth, (ii)

254 spoken at meetings organised by Wyeth and (iii) received sponsorship to travel to

255 congresses from Wyeth, as well as from numerous other pharmaceutical and

256 diagnostic companies. He holds shares in GlaxoSmithKline, Schering-Plough,

257 AstraZeneca, Dechra and Pfizer, who have now taken over Wyeth; he acts also as

258 Enduring Attorney for a close relative, managing further holdings in GlaxoSmithKline

259 and EcoAnimal Health. N. W. has received research grants from Wyeth. M. H., M. E.,

260 M. D., D. M. L. and N. W. are employees of the HPA and are influenced by its views

261 on antibiotic use and prescribing. G.S. has none to declare.

262 Ethical approval: Not required. 
1. Reinert RR, Low DE, Rossi F, Zhang X, Wattal C, Dowzicky MJ. Antimicrobial susceptibility among organisms from the Asia/Pacific Rim, Europe and Latin and North America collected as part of TEST and the in vitro activity of tigecycline. J Antimicrob Chemother 2007; 60: 1018-29.

2. Livermore DM, Hope R, Brick G, Lillie M, Reynolds R. Non-susceptibility trends among Enterobacteriaceae from bacteraemias in the UK and Ireland, 2001-06. J Antimicrob Chemother 2008; 62 Suppl 2: ii41-ii54.

3. Anthony KB, Fishman NO, Linkin DR, Gasink LB, Edelstein PH, Lautenbach

E. Clinical and microbiological outcomes of serious infections with multidrugresistant Gram-negative organisms treated with tigecycline. Clin Infect Dis 2008; 46: 567-70.

4. Keeney D, Ruzin A, Bradford PA. RamA, a Transcriptional regulator, and AcrAB, an RND-type efflux pump, are associated with decreased susceptibility to tigecycline in Enterobacter cloacae. Microb Drug Resist 2007; 13: 1-6.

5. Daurel C, Fiant AL, Bremont S, Courvalin P, Leclercq R. Emergence of an Enterobacter hormaechei with reduced susceptibility to tigecycline under tigecycline therapy. Antimicrob Agents Chemother 2009.

6. Andrews JM. BSAC standardized disc susceptibility testing method (version 8).

7. Sambrook J, Fritsch EF, Maniatis T. Molecular cloning: a laboratory Manual. 2nd. 1989. New York, Cold Spring Harbour Laboratory Press. 
8. Livak KJ, Schmittgen TD. Analysis of relative gene expression data using realtime quantitative PCR and the $2^{-\Delta \Delta C T}$ Method. Methods 2001; 25: 402-8.

9. Perez A, Canle D, Latasa C, Poza M, Beceiro A, del Mar Tomas M. et al. Cloning, nucleotide sequencing, and analysis of the AcrAB-TolC efflux pump of Enterobacter cloacae and determination of its involvement in antibiotic resistance in a clinical isolate. Antimicrob Agents Chemother 2007; 51: 3247 53.

10. Chaveroche MK, Ghigo JM, d'Enfert C. A rapid method for efficient gene replacement in the filamentous fungus Aspergillus nidulans. Nucleic Acids Res 2000; 28: E97. Four new derivatives of the broad-host-range cloning vector pBBR1MCS, carrying different antibiotic-resistance cassettes. Gene 1995; 166: 175-6. 
305

306

307

308

309

310

311

312

313

314 Figure 2

315 Relative expression (means \pm standard deviations) of acrAB in TGC-S harbouring

316 either control plasmid pBADKM (white) or pBADKM-R (black) in the presence of

317 varying concentrations of L-arabinose.

\section{Figure Legends}

Figure 1

PFGE profiles of clinical isolates TGC-S and TGC-R and all laboratory-selected mutants.

318 
Table 1. Characteristics of E. cloacae isolates and derivatives used in this study

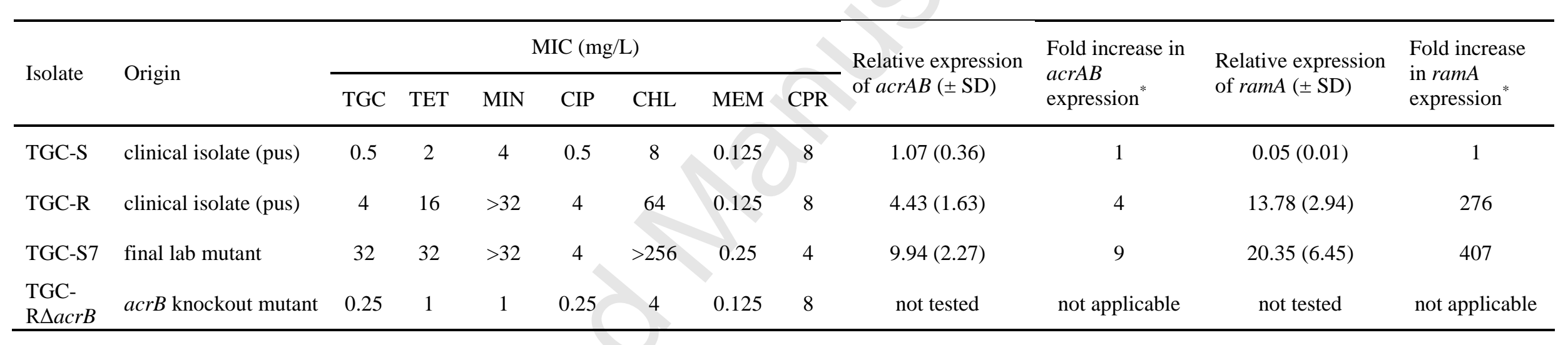

*relative to TGC-S

Antibiotic abbreviations: TGC, tigecycline; TET, tetracycline MIN, minocycline; CIP, ciprofloxacin; CHL, chloramphenicol; MEM, meropenem; CPR, cefpirome. 


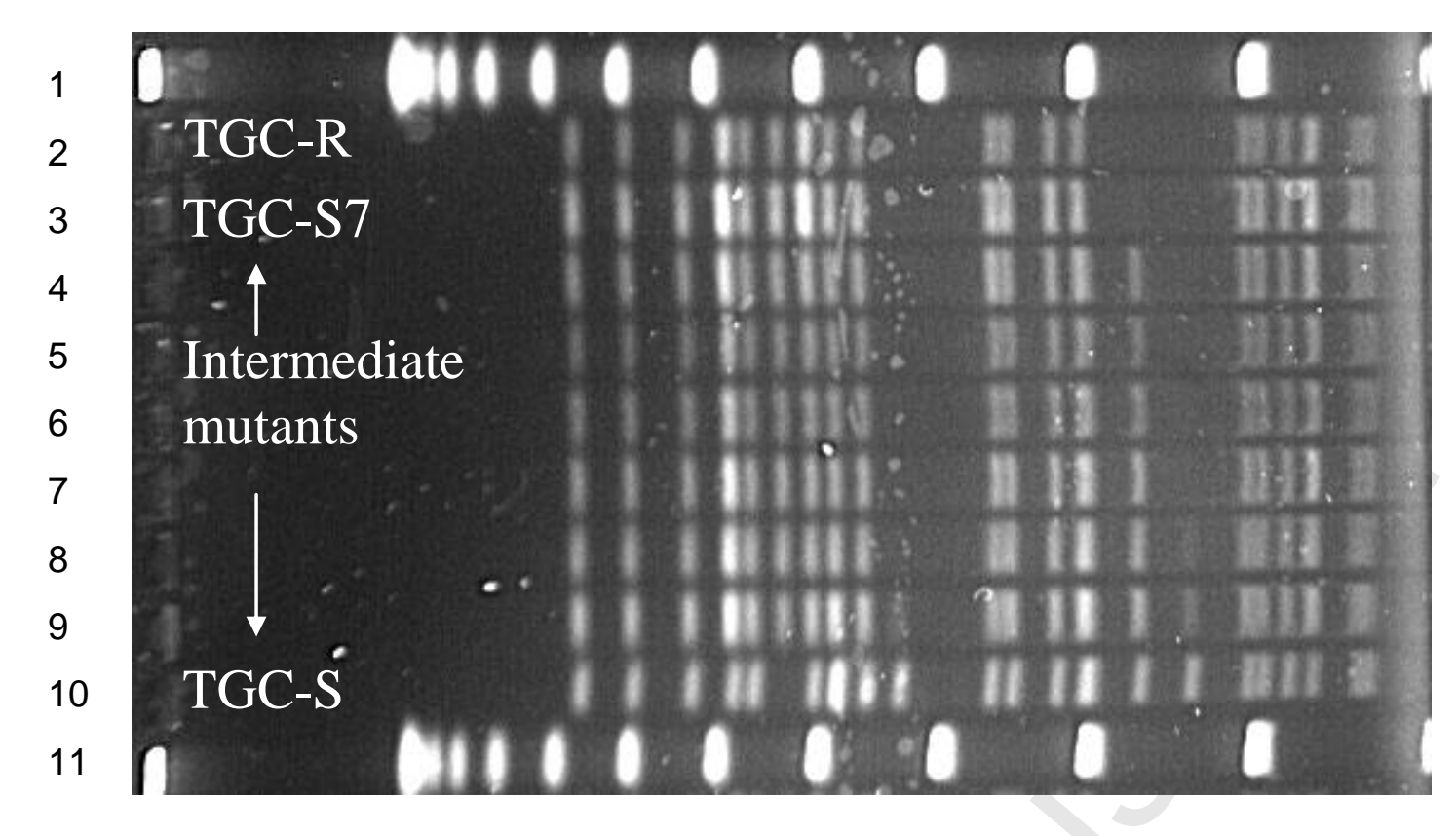

Figure 1.

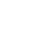

Figure 1. 
